\title{
openheart Risk prediction in patients with low- flow, low-gradient aortic stenosis and reduced ejection fraction undergoing TAVI
}

\author{
Sebastian Ludwig (D) , ${ }^{1,2}$ Alina Goßling, ${ }^{1}$ Moritz Seiffert, ${ }^{1,2}$ Dirk Westermann (D) , \\ Jan-Malte Sinning, ${ }^{3}$ Atsushi Sugiura, ${ }^{3}$ Matti Adam, ${ }^{4}$ Victor Mauri (D) , ${ }^{4}$ \\ Derk Frank (D) , ${ }^{5}$ Hatim Seoudy (D) , ${ }^{5}$ Tanja Rudolph, ${ }^{6}$ Max Potratz, ${ }^{6}$ \\ Lenard Conradi, ${ }^{2,7}$ Niklas Schofer ${ }^{1,2}$
}

\begin{abstract}
- Additional supplemental material is published online only. To view, please visit the journal online (http://dx.doi.org/10. 1136/openhrt-2021-001912).

To cite: Ludwig S, Goßling A, Seiffert M, et al. Risk prediction in patients with low-flow, lowgradient aortic stenosis and reduced ejection fraction undergoing TAVI. Open Heart 2022;9:e001912. doi:10.1136/ openhrt-2021-001912
\end{abstract}

Received 8 November 2021 Accepted 6 December 2021
Check for updates

(C) Author(s) (or their employer(s)) 2022. Re-use permitted under CC BY-NC. No commercial re-use. See rights and permissions. Published by BMJ.

For numbered affiliations see end of article.

Correspondence to Dr Sebastian Ludwig; se. ludwig@uke.de

\section{ABSTRACT}

Objective Patients with low-flow, low-gradient aortic stenosis (LFLG AS) and reduced left ventricular ejection fraction (LVEF) are known to suffer from poor prognosis after transcatheter aortic valve implantation (TAVI). This study aimed to develop a simple score system for risk prediction in this vulnerable subset of patients.

Methods All patients with LFLG AS with reduced EF and sufficient CT data for aortic valve calcification (AVC) quantification, who underwent TAVI at five German centres, were retrospectively included. The Risk prEdiction in patients with Low Ejection Fraction low gradient aortic stenosis undergoing TAVI (RELiEF TAVI) score was developed based on multivariable Cox regression for allcause mortality.

Results Among all included patients $(n=718)$, RELiEF TAVI score variables were defined as independent predictors of mortality: male sex (HR $1.34(1.06,1.68)$, $p=0.013)$, underweight (HR $3.10(1.50,6.40), p=0.0022)$, chronic obstructive pulmonary disease (HR 1.55 (1.21, 1.99), $p=0.001$ ), pulmonary hypertension (HR 1.51 (1.17, 1.94), $p=0.0015)$, atrial fibrillation (HR $1.28(1.03,1.60)$, $\mathrm{p}=0.028)$, stroke volume index (HR $0.96(0.95,0.98)$, $\mathrm{p}<0.001)$, non-transfemoral access (HR $1.36(1.05,1.76)$, $\mathrm{p}=0.021)$ and low AVC density (HR $1.44(1.15,1.79)$, $\mathrm{p}=0.0012$ ). A score system was developed ranging from 0 to 12 points (risk of 1-year mortality: 13\%-99\%). KaplanMeier analysis for low (0-1 points), moderate (2-4 points) and high RELIEF TAVI score ( $>4$ points) demonstrated rates of $18.0 \%, 29.0 \%$ and $46.1 \%(p<0.001)$ for all-cause mortality and $23.8 \%, 35.9 \%$ and $53.4 \%(p<0.001)$ for the combined endpoint of all-cause mortality or heart failure rehospitalisation after 1 year, respectively.

Conclusions The RELiEF TAVI score is based on simple clinical, echocardiographic and CT parameters and might serve as a helpful tool for risk prediction in patients with LFLG AS and reduced LVEF scheduled for TAVI.

\section{INTRODUCTION}

For the majority of patients with severe symptomatic aortic stenosis (AS), transcatheter aortic valve implantation (TAVI) has become

\section{Key questions}

What is already known about this subject?

- Patients with low-flow, low-gradient aortic stenosis (LFLG AS) and reduced left ventricular ejection fraction (LVEF) suffer from poor prognosis after transcatheter aortic valve implantation (TAVI), especially when compared with patients with high-gradient AS. However, data on strong predictors of outcome in this complex subset of patients are scarce.

What does this study add?

- Based on a large retrospective registry, the present study was able to define simple clinical, echocardiographic and CT parameters at baseline, that were independently associated with adverse outcome of patients with LFLG AS and reduced LVEF after TAVI. By integrating these parameters, the The Risk prEdiction in patients with Low Ejection Fraction low gradient aortic stenosis undergoing TAVI (RELiEF TAVI) score was developed as a tool for risk discrimination in patients with LFLG AS and reduced LVEF scheduled for intervention.

How might this impact on clinical practice?

- The RELiEF TAVI score consists of simple baseline clinical, echocardiographic and CT parameters and might help identify patients with acceptable outcome after TAVI within the vulnerable subset of patients with LFLG AS and reduced LVEF. Moreover, it might be used as a tool to identify those patients, in whom TAVI may be considered futile. Consequently, the RELiEF TAVI score may facilitate decisionmaking in patients with LFLG AS and reduced LVEF.

the standard therapy within recent years. ${ }^{1}$ According to the 2021 European Society of Cardiology/European Association for Cardio-Thoracic Surgery guidelines for the management of valvular heart disease, severe AS can be categorised into different subtypes with respect to the transvalvular gradient (Pmean), the flow status across the aortic 
valve (as assessed by stroke volume index (SVI)) and left ventricular ejection fraction (LVEF). ${ }^{2}$ While patients with high-gradient AS (Pmean $\geq 40 \mathrm{~mm} \mathrm{Hg}$ ) experience the largest benefit after TAVI, those with low-flow, lowgradient (LFLG) AS and reduced LVEF (Pmean $<40 \mathrm{~mm}$ $\mathrm{Hg}$, aortic valve area (AVA) $\leq 1 \mathrm{~cm}^{2}, \mathrm{LVEF}<50 \%$, SVI $\leq 35$ $\mathrm{mL} / \mathrm{m}^{2}$ ) have a markedly poorer prognosis with mortality rates of $30 \%-50 \%$ or higher already at 2 years after treatment. ${ }^{3-6}$ Consequently, clinical decision-making in these patients is challenging because strong predictors of outcome following TAVI for this subset of patients have not yet been adequately determined.

The aim of the current study was to develop a simple score system for risk prediction based on clinical, echocardiographic and CT parameters at baseline derived from a large multicentre cohort of patients with LFLG AS and reduced LVEF undergoing TAVI (Risk prEdiction in patients with Low Ejection Fraction low gradient aortic stenosis undergoing TAVI (RELiEF TAVI) score).

\section{MATERIAL AND METHODS}

\section{Study design and data acquisition}

The study was designed as a retrospective analysis of data derived from five German high-volume TAVI centres. All clinical endpoints were adjudicated according to the Valve Academic Research Consortium-2 (VARC-2) criteria after 30 days. Echocardiographic outcome was derived from in-house transthoracic echocardiography (TTE) at discharge. Survival data were obtained from in-house information as part of clinical routine follow-up. All patients provided informed consent to the procedure and data acquisition. It was not appropriate or possible to involve patients or the public in the design, or conduct, or reporting, or dissemination plans of our research. The study was approved by the local institutional review boards and performed in accordance with the declaration of Helsinki.

\section{Study population}

Between 2008 and 2019, a total of 9150 patients were treated with TAVI at all participating TAVI centres. For all patients, the decision to perform TAVI was made by an interdisciplinary heart team. After excluding patients who underwent planned valve-in-valve procedures, combined percutaneous mitral valve treatment, patients treated with investigational transcatheter heart valves (THVs) and those with insufficient CT data for the quantification of aortic valve calcification (AVC), we identified 718 patients fulfilling the echocardiographic criteria for severe LFLG AS with reduced LVEF.

\section{Study endpoints}

The primary endpoint was defined as all-cause mortality after 1 year. Secondary endpoints were cardiovascular mortality and a combined endpoint of all-cause mortality or heart failure (HF) rehospitalisation after 1 year.

\section{Definition of LFLG AS with reduced ejection fraction}

Severe LFLG AS with reduced LVEF was defined as an AVA $\leq 1.0 \mathrm{~cm}^{2}$, transvalvular gradient $<40 \mathrm{~mm} \mathrm{Hg}$, SVI $\leq 35 \mathrm{~mL} / \mathrm{m}^{2}$ and $\mathrm{LVEF}<50 \%$ assessed by means of resting TTE. AVAs were calculated by using the continuity equation. ${ }^{7}$ SVI was calculated by means of cross-sectional left ventricular outflow tract (LVOT) area and LVOT velocity time integral. ${ }^{2}$

\section{CT measurements}

Quantification of AVC was performed by calcium volume scoring on contrast-enhanced CT images using a dedicated software (3mensio Structural Heart V9.1, Pie Medical Imaging, Maastricht, Netherlands). An empirical threshold of 550 Hounsfield units (HU) was used for AVC assessment in the majority of patients for the purpose of discrimination between calcium and contrast medium. The threshold was only adjusted in exceptional cases in order to properly distinguish calcium from contrast agent. Calcification was separately assessed in the annular plane and the LVOT, as described before. ${ }^{8}$ AVC was defined as the total calcium volume detected in the annular plane sector. AVC density (AVCd) was defined as the ratio of AVC $\left(\mathrm{mm}^{3}\right.$ calcium) per aortic annulus area $\left(\mathrm{cm}^{2}\right)$. Distribution of AVCd measurements for each centre is demonstrated by density curves in online supplemental figure S1. Low AVCd was defined as the lowest sex-specific AVCd tertile in the derivation cohort, that is, AVCd $\leq 62.45 \mathrm{~mm}^{3}$ calcium $/ \mathrm{cm}^{2}$ for male patients and AVCd $\leq 51.04 \mathrm{~mm}^{3}$ calcium $/ \mathrm{cm}^{2}$ for female patients. Calcification of the LVOT was assessed separately. Low LVOT calcification was defined as LVOT calcium load $\leq 10$ $\mathrm{mm}^{3}$, as described before. ${ }^{9}$

\section{Statistical analysis}

Sex-specific AVCd tertiles were calculated in the overall study population. Boundaries for sex-specific AVCd tertiles are given in online supplemental table S1. Variables entering the score system were selected in a two-step selection process. Variables that showed $p$ values $<0.1$ in the univariable Cox regression analysis (1) were used in a forward selection process based on Akaike Information Criteria (2). The variables of the best performing multivariable model were used for risk score calculation. In order to assign risk points to the selected variables and to predict mortality rates by risk score points, we followed the method used by the Framingham Heart Study. One score point was reflected by 0.29 regression units. Underweight was defined as body mass index $(\mathrm{BMI})<18.5 \mathrm{~kg} / \mathrm{m}^{2}{ }^{10}$

For validation, a bootstrap correction with 500 bootstraps was performed. Survival curves were produced using the Kaplan-Meier method and curve differences were tested using the log-rank test. Model performance was assessed by calibration and discrimination. The c-index for all endpoints was calculated for the RELiEF TAVI score and for an established risk score system, the European System for Cardiac Operative Risk Evaluation (EuroSCORE) II. ${ }^{11}$ All statistical analyses were performed 
Table 1 Baseline characteristics, procedural aspects and outcomes

\section{Study population $(n=718)$}

Clinical baseline characteristics

\begin{tabular}{|c|c|}
\hline Age (years) & $81.0(76.4,84.7)$ \\
\hline Male sex & $448(62.4)$ \\
\hline $\mathrm{BMI}\left(\mathrm{kg} / \mathrm{m}^{2}\right)$ & $25.8(23.5,29.4)$ \\
\hline EuroSCORE II (\%) & $7.0(4.1,12.4)$ \\
\hline COPD & $177(24.7)$ \\
\hline Diabetes & $240(33.4)$ \\
\hline Atrial fibrillation & $382(53.7)$ \\
\hline Extracardiac arteriopathy & $277(38.6)$ \\
\hline $\begin{array}{l}\text { Impaired renal function (GFR }<50 \mathrm{~mL} \text { / } \\
\mathrm{min} / 1.73 \mathrm{~m}^{2} \text { ) }\end{array}$ & $351(49.0)$ \\
\hline Prior myocardial infarction & $226(31.5)$ \\
\hline Prior stroke & $128(17.8)$ \\
\hline Prior CABG & $130(22.8)$ \\
\hline NYHA functional class III & $435(66.2)$ \\
\hline NYHA functional class IV & $141(21.5)$ \\
\hline \multicolumn{2}{|l|}{ Echocardiographic parameters } \\
\hline $\mathrm{P}$ mean $(\mathrm{mm} \mathrm{Hg})$ & $25.0(19.0,31.0)$ \\
\hline $\mathrm{SVI}\left(\mathrm{mL} / \mathrm{m}^{2}\right)$ & $27.1(23.1,31.0)$ \\
\hline $\operatorname{AVA}\left(\mathrm{cm}^{2}\right)$ & $0.8(0.6,0.9)$ \\
\hline Indexed EOA $\left(\mathrm{cm}^{2} / \mathrm{m}^{2}\right)$ & $0.4(0.3,0.5)$ \\
\hline LVEF $<30 \%$ & $217(30.2)$ \\
\hline Pulmonary hypertension (sPAP >55 mm Hg) & $131(23.4)$ \\
\hline$\geq$ Moderate MR & $316(44.3)$ \\
\hline$\geq$ Moderate TR & $252(36.2)$ \\
\hline \multicolumn{2}{|l|}{ CT parameters } \\
\hline Perimeter derived diameter (mm) & $25.4(23.8,26.8)$ \\
\hline Annulus area $\left(\mathrm{mm}^{2}\right)$ & $495.4(436.5,557.5)$ \\
\hline AVC (mm³ calcium) & $392.1(235.1,672.0)$ \\
\hline AVCd $\left(\mathrm{mm}^{3}\right.$ calcium $\left./ \mathrm{cm}^{2}\right)$ & $80.8(48.6,133.6)$ \\
\hline LVOT calcification ( $\mathrm{mm}^{3}$ calcium) & $1.4(0,29.7)$ \\
\hline$\leq 10 \mathrm{~mm}^{3}$ LVOT calcium & $460(64.2)$ \\
\hline \multicolumn{2}{|l|}{ Procedural characteristics } \\
\hline TF access & $597(83.1)$ \\
\hline Non-TF access & $121(16.9)$ \\
\hline TA access & $85(11.8)$ \\
\hline TA0 access & $14(1.9)$ \\
\hline TAX access & $22(3.1)$ \\
\hline Balloon-expandable THV & $294(40.9)$ \\
\hline Self-expandable THV & $401(55.8)$ \\
\hline Mechanically expandable THV & $16(2.2)$ \\
\hline Non-metallic THV & $7(1.0)$ \\
\hline \multicolumn{2}{|l|}{ 30-day VARC-2 outcomes } \\
\hline Disabling stroke & $19(2.6)$ \\
\hline
\end{tabular}

Table 1 Continued

\section{Study population $(n=718)$}

\begin{tabular}{|c|c|}
\hline New permanent pacemaker implantation & $97(13.6)$ \\
\hline Major/life-threatening bleeding & $68(9.5)$ \\
\hline Acute renal failure (AKIN $\geq 2$ ) & $18(4.7)$ \\
\hline$\geq$ Moderate PVL & $29(4.3)$ \\
\hline \multicolumn{2}{|l|}{ Mortality } \\
\hline Procedural mortality (\%) & 1.1 \\
\hline 30-day mortality (\%) & 7.7 \\
\hline 1-year mortality (\%) & 29.1 \\
\hline
\end{tabular}

Data presented are the number (\%) of patients for categorical variables or median values (25th percentile, 75 th percentile) for continuous variables.

AKIN, acute kidney injury network; AVA, aortic valve area; AVC, aortic valve calcification; AVCd, aortic valve calcification density; $\mathrm{BMI}$, body mass index; CABG, coronary artery bypass graft; COPD, chronic obstructive pulmonary disease; EOA, effective orifice area; EuroSCORE II, European System for Cardiac Operative Risk Evaluation II; GFR, glomerular filtration rate; LVEF, left ventricular ejection fraction; LVOT, left ventricular outflow tract; P mean, mean transvalvular pressure gradient; MR, mitral regurgitation; NYHA, New York Heart Association; PVL, paravalvular leakage; sPAP, systolic pulmonary artery pressure; SVI, stroke volume index; TA, transapical; TAO, transaortic; TAX, transaxillary; TF, transfemoral; THV, transcatheter heart valve; $\mathrm{TR}$, tricuspid regurgitation; VARC-2, valve academic research consortium-2.

using R V.4.0.3 (R Foundation for Statistical Computing, Vienna, Austria).

\section{RESULTS}

\section{Baseline characteristics and outcomes}

Clinical, echocardiographic and CT baseline parameters as well as procedural characteristics and outcome data of the overall study population $(\mathrm{n}=718)$ are given in table 1 . The investigated patients were predominantly men and of advanced age with a median of 81.0 years (IQR 76.4, 84.7). Cardiovascular comorbidities (ie, atrial fibrillation, severe pulmonary hypertension, prior myocardial infarction, prior stroke or extracardiac arteriopathy) and non-cardiovascular comorbidities (ie, chronic obstructive pulmonary disease (COPD), diabetes or impaired renal function) were present in a large proportion of the study population, translating into elevated surgical risk (EuroSCORE II $7.0 \%$ (IQR 4.1, 12.4)). Of note, $21.5 \%$ of the overall study population presented with New York Heart Association (NYHA) functional class IV. The majority of patients was treated via transfemoral access $(83.1 \%)$ with either balloon-expandable $(40.9 \%)$ or selfexpandable (55.8\%) THVs. Additional procedural data and outcomes are given in online supplemental table S2.

\section{0-Day VARC-2 and late clinical outcomes}

Within 30 days after TAVI, disabling stroke, major or lifethreatening bleeding, need for new pacemaker implantation or $\geq$ moderate paravalvular leakage occurred in 
Table 2 Univariable and multivariable Cox proportional regression analysis for all-cause mortality and RELiEF TAVI score development

\begin{tabular}{|c|c|c|c|c|c|c|}
\hline \multicolumn{5}{|c|}{ Univariate and multivariate Cox proportional regression analysis $(n=718)$} & \multicolumn{2}{|c|}{ RELiEF TAVI score } \\
\hline & \multicolumn{2}{|c|}{ Univariable analysis } & \multicolumn{2}{|c|}{ Multivariable analysis $(n=673)$} & \multirow[b]{2}{*}{$\boldsymbol{\beta}$} & \multirow{2}{*}{$\begin{array}{l}\text { Resulting } \\
\text { points }\end{array}$} \\
\hline & HR (95\% Cl) & $P$ value & HR (95\% Cl) & P value & & \\
\hline \multicolumn{7}{|l|}{ Clinical parameters } \\
\hline Age & 1.00 (0.99 to 1.02$)$ & 0.53 & & & & \\
\hline Male sex & 1.38 (1.11 to 1.71$)$ & 0.0036 & 1.34 (1.06 to 1.68$)$ & 0.013 & 1.00 & 1 \\
\hline $\begin{array}{l}\text { Underweight }(\mathrm{BMI}<18.5 \\
\left.\mathrm{kg} / \mathrm{m}^{2}\right)\end{array}$ & 2.86 (1.45 to 5.64$)$ & 0.0025 & 3.10 (1.50 to 6.40$)$ & 0.0022 & 3.90 & 4 \\
\hline GFR $<60 \mathrm{~mL} / \mathrm{min}$ & 1.19 (0.95 to 1.48$)$ & 0.13 & & & & \\
\hline Diabetes & $1.08(0.87$ to 1.35$)$ & 0.46 & & & & \\
\hline COPD & 1.54 (1.23 to 1.95$)$ & $<0.001$ & 1.55 (1.21 to 1.99 ) & $<0.001$ & 1.52 & 2 \\
\hline Atrial fibrillation & 1.42 (1.15 to 1.75$)$ & 0.0011 & 1.28 (1.03 to 1.60$)$ & 0.028 & 0.86 & 1 \\
\hline Prior stroke & 1.21 (0.93 to 1.57 ) & 0.16 & & & & \\
\hline Prior myocardial infarction & 1.07 (0.85 to 1.35$)$ & 0.55 & & & & \\
\hline \multicolumn{7}{|l|}{ Echocardiography } \\
\hline SVI & 0.96 (0.95 to 0.98$)$ & $<0.001$ & 0.96 (0.95 to 0.98$)$ & $<0.001$ & $1.27^{\star}$ & 1 \\
\hline \multicolumn{7}{|l|}{ LVEF } \\
\hline LVEF $45 \%-49 \%$ & 1 (reference) & & & & & \\
\hline LVEF $30 \%-44 \%$ & 1.21 (0.93 to 1.57 ) & 0.16 & & & & \\
\hline LVEF $<30 \%$ & 1.42 (1.09 to 1.84 ) & 0.0095 & & & & \\
\hline$\geq$ Moderate TR & 1.33 (1.08 to 1.65$)$ & 0.0085 & & & & \\
\hline$\geq$ Moderate MR & 1.00 (0.81 to 1.23$)$ & 0.99 & & & & \\
\hline $\begin{array}{l}\text { Pulmonary hypertension } \\
(\text { (SPAP }>55 \mathrm{~mm} \mathrm{Hg})\end{array}$ & 1.53 (1.19 to 1.96$)$ & $<0.001$ & 1.51 (1.17 to 1.94$)$ & 0.0015 & 1.42 & 1 \\
\hline \multicolumn{7}{|l|}{ CT } \\
\hline Non-TF access & 1.40 (1.10 to 1.80$)$ & 0.0071 & 1.36 (1.05 to 1.76$)$ & 0.021 & 1.05 & 1 \\
\hline Low AVCd $†$ & 1.33 (1.08 to 1.65$)$ & 0.0081 & 1.44 (1.15 to 1.79$)$ & 0.0012 & 1.25 & 1 \\
\hline$\leq 10 \mathrm{~mm}^{3}$ LVOT calcium & 1.16 (0.92 to 1.45$)$ & 0.21 & & & & \\
\hline
\end{tabular}

Abbreviations as in table 1.

*Statistically derived cut-off: SVI $\leq 24.9 \mathrm{~mL} / \mathrm{m}^{2}$.

†Male cut-off: $\leq 62.45 \mathrm{~mm}^{3}$ calcium $/ \mathrm{cm}^{2}$, female cut-off: $\leq 51.04 \mathrm{~mm}^{3}$ calcium $/ \mathrm{cm}^{2}$.

AVCd, aortic valve calcification density; BMI, body mass index; COPD, chronic obstructive pulmonary disease; GFR, glomerular filtration rate; LVEF, left ventricular ejection fraction; LVOT, left ventricular outflow tract; MR, mitral regurgitation; RELiEF TAVI, Risk prEdiction in patients with Low Ejection Fraction low gradient aortic stenosis undergoing TAVI; sPAP, systolic pulmonary artery pressure; SVI, stroke volume index; TAVI, transcatheter aortic valve implantation; TF, transfemoral; TR, tricuspid regurgitation.

$2.6 \%, 9.5 \%, 13.6 \%$ and $4.3 \%$ of the patients. Procedural and 30 -day mortality rates were $1.1 \%$ and $7.7 \%$, respectively (table 1). Over the median follow-up time of 2.95 (95\% CI 2.66 to 3.21 ) years, death occurred in 371 cases. Cardiovascular death and HF rehospitalisation occurred in 84 and 126 patients, respectively. Among the study population all-cause mortality rate 1 year after TAVI was $29.1 \%$.

\section{RELIEF TAVI score development}

Uni- and multivariable Cox regression analyses for allcause mortality in the total patient population as well as score point assignment are given in table 2. After multivariable analysis male sex (HR 1.34 (95\% confidence interval (CI): 1.06 to 1.68$), \mathrm{p}=0.013$ ), underweight (HR
3.10 (95\% CI: 1.50 to 6.40 ), $\mathrm{p}=0.0022$ ), COPD (HR 1.55 (95\% CI: 1.21 to 1.99$), \mathrm{p}<0.001)$, pulmonary hypertension $>55 \mathrm{~mm} \mathrm{Hg}$ (HR 1.51 (95\% CI: 1.17 to 1.94), $\mathrm{p}=0.0015)$, atrial fibrillation (HR 1.28 (95\% CI: 1.03 to $1.60), \mathrm{p}=0.028)$, non-transfemoral access (HR $1.36(95 \%$ CI: 1.05 to 1.76$), \mathrm{p}=0.021)$ and low AVCd (HR $1.44(95 \%$ CI: 1.15 to 1.79$), \mathrm{p}=0.0012)$ remained independent predictors of mortality, while SVI (continuous) (HR 0.96 (95\% CI: 0.95 to 0.98$), \mathrm{p}<0.001$ ) was independently predictive of survival. Figure 1 shows a forest plot for all independent predictors of outcome.

All parameters included in this multivariable Cox regression model were selected as score variables and, according to their predictive weight, score points were 


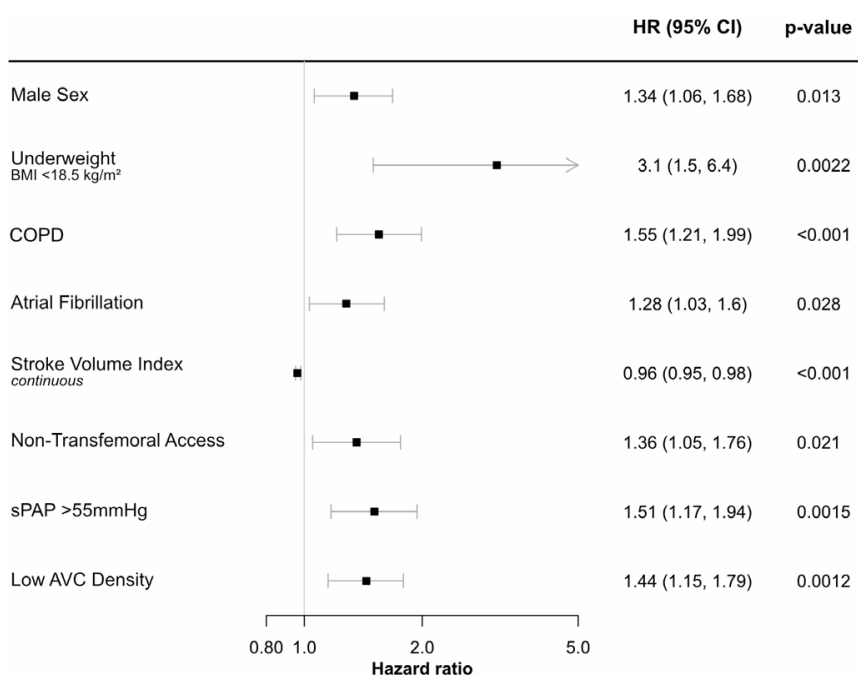

Figure 1 Forest plot for all independent predictors of outcome. HRs for all-cause mortality after multivariable adjustment. AVC, aortic valve calcification; BMI, body mass index; COPD, chronic obstructive pulmonary disease; SPAP, systolic pulmonary artery pressure. assigned to each score variable: Male sex (one point), COPD (two points), atrial fibrillation (one point), underweight (BMI $<18.5 \mathrm{~kg} / \mathrm{m}^{2}$ ) (four points), low SVI ( $\leq 24.9$ $\mathrm{mL} / \mathrm{m}^{2}$ ) (one point), pulmonary hypertension $>55 \mathrm{~mm}$ $\mathrm{Hg}$ (one point), low AVCd (male: $\leq 62.45 \mathrm{~mm}^{3}$ calcium/ $\mathrm{cm}^{2}$; female: $\leq 51.04 \mathrm{~mm}^{3}$ calcium $/ \mathrm{cm}^{2}$ ) (one point) and non-transfemoral access (one point). The RELiEF TAVI score is summarised in figure 2 and ranges from 0 (estimated risk of 1-year mortality: $13 \%$ ) to 12 points (estimated risk of 1-year mortality: 99\%). Online supplemental figure S2 depicts the distribution of score points across the study population.

\section{Risk discrimination}

Figure 3 demonstrates predicted mortality by the RELiEF TAVI score and observed mortality for each score point in the study population. While observed 1-year mortality in study patients with 0 points was $22.4 \%$, mortality after 1 year reached $92.3 \%$ in study patients with 7 or more RELiEF TAVI score points. All patients with more than 7 points died within 1 year after TAVI.

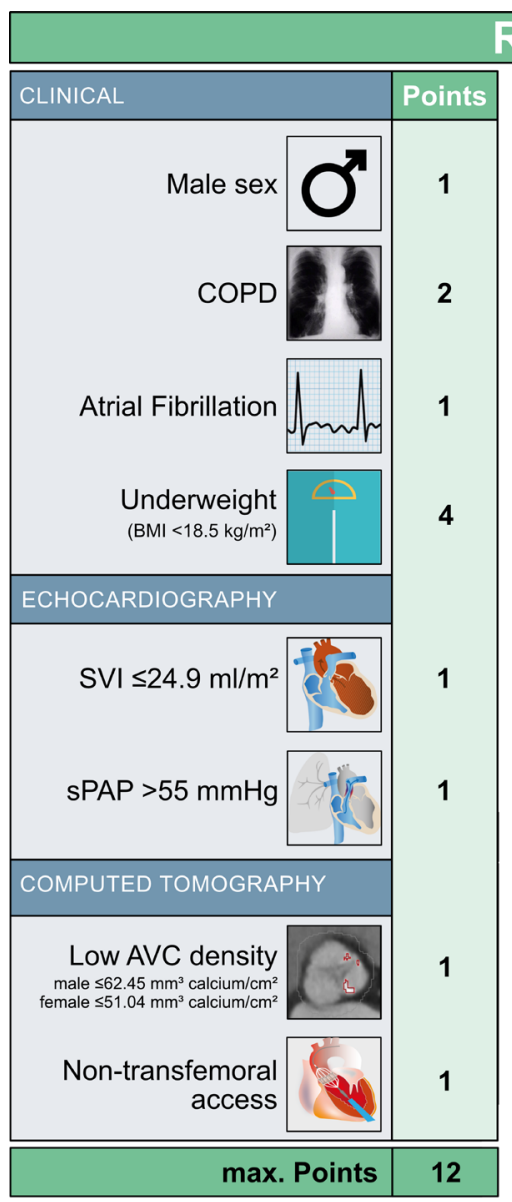

\section{RELLIEF TAVI Score}
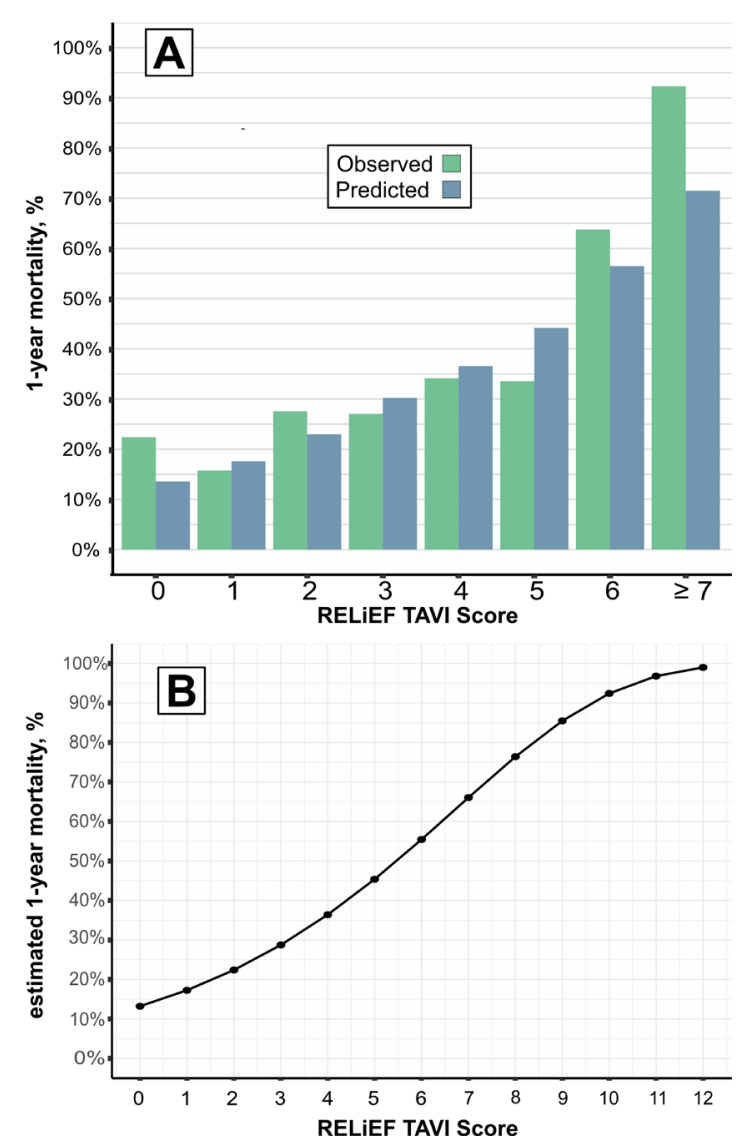

Figure 2 The RELiEF TAVI score. Variables, score point assignment and prediction of 1-year all-cause mortality risk. AVC, aortic valve calcification; BMI, body mass index; COPD, chronic obstructive pulmonary disease; RELiEF TAVI, Risk prEdiction in patients with Low Ejection Fraction low gradient aortic stenosis undergoing TAVI; sPAP, systolic pulmonary artery pressure; SVI, stroke volume index; TAVI, transcatheter aortic valve implantation. 


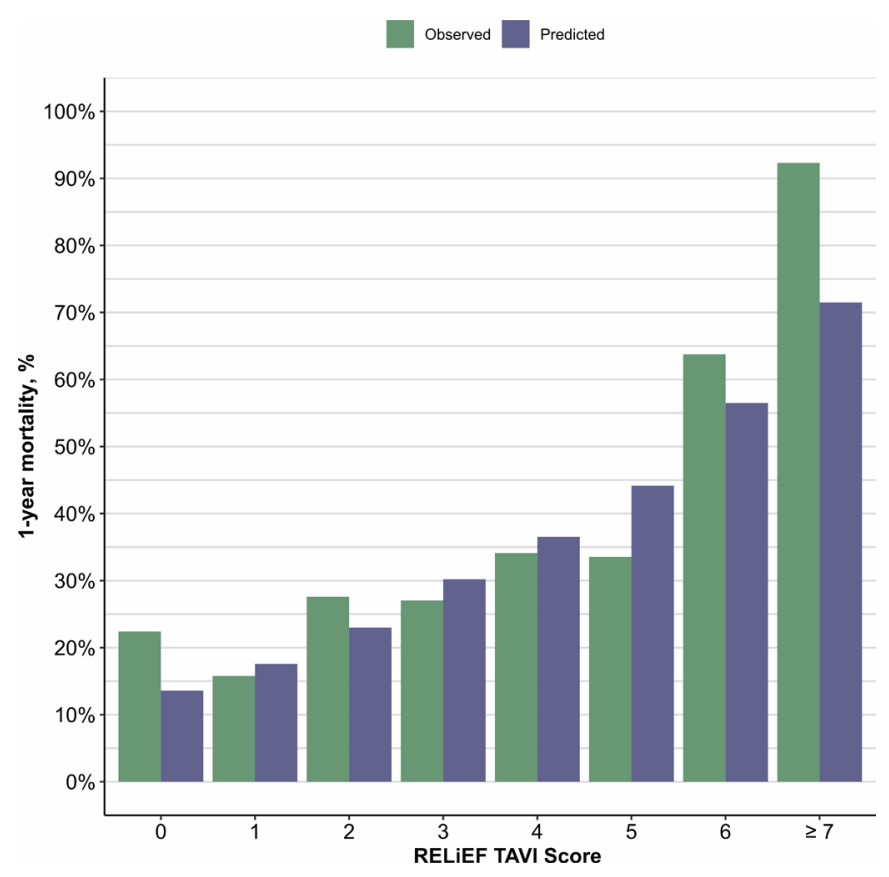

Figure 3 Observed mortality and predicted 1-year allcause mortality by the RELiEF TAVI score. RELiEF TAVI, Risk prEdiction in patients with Low Ejection Fraction low gradient aortic stenosis undergoing TAVI; TAVI, transcatheter aortic valve implantation.

Kaplan-Meier analyses for the primary endpoint of all-cause mortality after 1 year as well as the secondary endpoint of cardiovascular mortality after 1 year and the combined endpoint of all-cause mortality or HF rehospitalisation after 1 year are given in figure 4 according to low (0-1 points), moderate (2-4 points) and high RELiEF TAVI score ( $>4$ points).

Regarding the primary endpoint, patients with high RELiEFTAVI score had the highest 1-year all-cause mortality rate compared with patients with moderate or low RELiEF TAVI score $(46.1 \%$ vs $29.0 \%$ vs $18.0 \%, \mathrm{p}<0.001)$. Log-rank tests for post hoc pairwise comparisons also demonstrated significant differences in mortality rates between low versus moderate RELiEF TAVI score $(\mathrm{p}=0.0058)$, moderate versus high RELiEF TAVI score $(\mathrm{p}<0.001)$ and low versus high RELiEF TAVI score $(\mathrm{p}<0.001)$ (figure $4 \mathrm{~A})$.

The secondary endpoint of cardiovascular mortality after 1 year and the combined endpoint of all-cause mortality or HF rehospitalisation after 1 year also occurred significantly more often in patients with high RELiEF TAVI score $(29.3 \%$ and $53.4 \%)$ compared with patients with moderate $(16.2 \%$ and $35.9 \%)$ or low $(7.0 \%$ and $23.8 \%)$ RELiEF TAVI score $(\mathrm{p}<0.001$ for both endpoints) (figure 4B,C).

\section{Risk prediction}

The c-index of the RELiEF TAVI score was 0.62 (95\% CI: 0.59 to 0.68 ) for all-cause mortality, 0.66 (95\% CI: 0.61 to 0.75 ) for cardiovascular mortality, 0.64 (95\% CI: 0.55 to $0.70)$ for $\mathrm{HF}$ rehospitalisation and 0.61 (95\% CI: 0.57 to 0.66 ) for the combined endpoint of all-cause mortality or HF rehospitalisation, all after 1 year. After internal bootstrap validation, c-indices of the RELiEF TAVI score were 0.60 (95\% CI: 0.57 to 0.66 ) for all-cause mortality, 0.64 (95\% CI: 0.59 to 0.73 ) for cardiovascular mortality, 0.63 (95\% CI: 0.54 to 0.59 ) for HF rehospitalisation and 0.59 (95\% CI: 0.56 to 0.64 ) for the combined endpoint after 1 year. Compared with the established EuroSCORE II, the RELiEF TAVI score was superior regarding the prediction of all-cause mortality (EuroSCORE II: 0.57 (95\% CI: 0.52 to 0.61$)$ ), HF rehospitalisation (EuroSCORE II: 0.58 (95\% CI: 0.48 to 0.66$)$ ) and the combined endpoint (EuroSCORE II: 0.56 (95\% CI: 0.53 to 0.60$)$ ), but not regarding cardiovascular mortality (EuroSCORE II: 0.70 (95\% CI: 0.63 to 0.77$)$ ).

\section{DISCUSSION}

In the present study, which comprises a large multicentre dataset of patients with LFLG AS and reduced LVEF
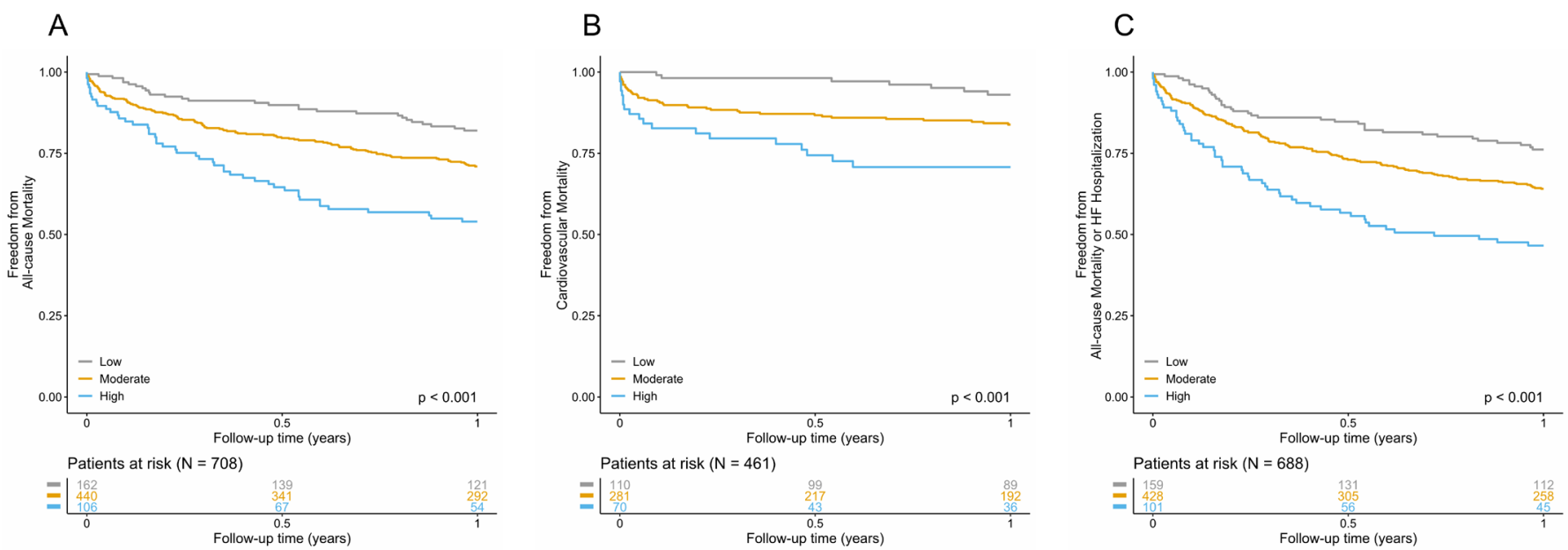

Figure 4 Kaplan-Meier analysis for different study endpoints according to low (0-1 points), moderate (2-4 points) and high RELiEF TAVI score (>4 points). (A) 1-year all-cause mortality, (B) 1-year cardiovascular mortality, (C) 1-year all-cause mortality or HF hospitalisation. HF, heart failure; RELiEF TAVI, Risk prEdiction in patients with Low Ejection Fraction low gradient aortic stenosis undergoing TAVI; TAVI, transcatheter aortic valve implantation. 
undergoing TAVI, a simple risk score system (RELiEF TAVI score) based on clinical, echocardiographic and CT parameters was developed and internally validated.

\section{Characteristics of patients with LFLG AS and reduced LVEF undergoing TAVI}

The limited prognosis of TAVI patients with LFLG AS and reduced LVEF has been shown in several previous studies. According to these data, patients presenting with LFLG AS and reduced LVEF suffer from a vast burden of cardiac as well as non-cardiac comorbidities. ${ }^{3-6}$ This is also demonstrated in the present study as a large proportion of patients presented with LVEF $<30 \%$, NYHA functional class IV, prior myocardial infarction, atrial fibrillation, concomitant mitral and tricuspid regurgitation, pulmonary hypertension, diabetes mellitus as well as peripheral artery and pulmonary disease. Although the rate of device success was high and procedural complication rates were low, all-cause mortality at 30 days and 12 months in the current study was still high with $7.3 \%$ and $29.1 \%$, respectively. Thus, in contrast to the universal trend to perform TAVI in patients with AS at lower risk, the subset of patients with LFLG AS and reduced LVEF clearly remains a high-risk population, in whomcompared with patients with non-LFLG AS-excess mortality after TAVI has to be anticipated. ${ }^{12} 13$

\section{Outcome predictors in patients with LFLG AS and reduced LVEF undergoing TAVI}

Outcome predictors among TAVI patients with LFLG AS and reduced LVEF have so far been assessed in national and international registries. By using data from the German Aortic Valve Registry, a prospective national registry including a total of 359 patients with LFLG AS and reduced LVEF that underwent TAVI, Lauten et al found peripheral artery disease, pulmonary hypertension and prior myocardial infarction to be independent predictors of mortality among patients with LFLG AS and reduced LVEF. ${ }^{5}$ The FRANCE 2 registry is a French national TAVI registry, that included 502 patients with LFLG AS and reduced LVEF. Amabile et al found that transapical access, pre-TAVI angina, advanced kidney failure, atrial fibrillation and moderate to severe aortic regurgitation after TAVI were independent outcome predictors in patients with LFLG AS and reduced LVEF included in the FRANCE 2 registry. ${ }^{14}$ In contrast to our study, in both national registries, SVI was not integrated in LFLG AS definition and neither CT nor dobutamine stress echocardiography (DSE) data were available for analysis. Moreover, the number of patients with LFLG AS and reduced LVEF was substantially higher in the present study $(\mathrm{n}=718)$. The True or Pseudo-severe Aortic Stenosis (TOPAS) registry is a prospective multicentric study including a total of 287 patients with LFLG AS and reduced LVEF undergoing TAVI. Of these, 234 patients underwent DSE prior to TAVI. Independent outcome predictors after TAVI in the TOPAS registry were COPD and low haemoglobin levels. ${ }^{3}$ Substudies of the TOPAS study identified right ventricular dysfunction and tricuspid regurgitation as important factors limiting prognosis of patients with LFLG AS and reduced LVEF. ${ }^{15} 16$ The present study, however, comprises a larger patient cohort compared with the TOPAS registry and represents the first analysis including both, SVI and AVC burden as assessed by CT. Beside male sex, we identified COPD, underweight, atrial fibrillation, pulmonary hypertension, non-transfemoral access, low AVCd and low SVI to be independently associated with mortality. The adverse impact of each of these variables on outcomes in the general TAVI population has been well described. ${ }^{3} 8$ 17-22 Low SVI, atrial fibrillation, pulmonary hypertension and COPD may be interpreted as surrogates for advanced chronic cardiac and non-cardiac illness, whereas nontransfemoral access is a marker for increased procedural risk. Underweight in elderly patients is a known surrogate parameter for frailty and has been associated with inferior outcome. ${ }^{23}$ In contrast, AVCd is a diagnostic parameter, which strongly correlates with AS severity. ${ }^{24}{ }^{25}$ Since it is completely independent from transvalvular flow, its diagnostic value has been described particularly for patients with LFLG AS and reduced LVEF. ${ }^{26}{ }^{27}$ In a study investigating outcomes of medically managed patients with severe AS, high AVCd was independently associated with excess mortality. ${ }^{28}$ Conversely, in a recent publication of patients with LFLG AS and reduced LVEF treated with TAVI, we showed that patients with high AVCd experience larger benefits compared with patients with low AVCd. ${ }^{8}$ Hence, the therapeutic benefit of eliminating AS with TAVI might be less pronounced in patients with low AVCd. The present study confirms the diagnostic and prognostic importance of CT based AVC assessment in patients with LFLG AS and reduced LVEF.

\section{Clinical value of the RELiEF TAVI score}

Based on simple clinical, echocardiographic and CT baseline parameters, we developed a new score system for the prediction of outcome in patients with LFLG AS and reduced LVEF scheduled for TAVI. In the present study, patients with LFLG AS and reduced LVEF with high RELiEF TAVI score ( $>4$ points) had a more than twofold increase in all-cause mortality or HF hospitalisations, a more than 2.5-fold increase in all-cause mortality alone and a fourfold increase in cardiovascular mortality at 1 year after TAVI compared with those with low RELiEF TAVI score (0-1 points). Moreover, the RELiEF TAVI score was superior the established EuroSCORE II regarding risk prediction of all-cause mortality and $\mathrm{HF}$ readmissions and provided a reasonable discriminative performance with only minor discrepancies between score-predicted and observed mortality rates. Given these results, the RELiEF TAVI score may serve as an additional tool to guide clinical decision-making in patients with LFLG AS and reduced LVEF. An important limitation of the present study is that we cannot provide data on medically treated patients with LFLG AS, which would allow for a comparison between TAVI and the natural course 
of AS in this high-risk patient population. Yet, in patients with a RELiEF TAVI score 7 or more, accounting for less than $2 \%$ of the total study cohort, the mortality rate 1 year after TAVI was in excess of $90 \%$. In such patients, who are at advanced stages of cardiac and non-cardiac comorbidities and present with only borderline AS according to CT (eg, according to guideline-supported thresholds (noncontrast CT) or, if unavailable, according to presented AVCd tertiles (contrast-enhanced CT)), treatment with TAVI may be considered futile, since these patients are very unlikely to experience prognostic benefit from the procedure. The majority of patients with LFLG AS and reduced LVEF, however, presents with low $(22.9 \%)$ or moderate RELiEF TAVI score (62.2\%), has a markedly better prognosis and, thus, is more likely to benefit from TAVI. This assumption is supported by data from a post hoc analysis of the randomised Partner A trial showing that TAVI improves outcome in the overall patient cohort with LFLG AS and reduced LVEF compared with medical treatment alone. ${ }^{4}$ Moreover, the ongoing TAVR UNLOAD randomised controlled trial (NCT02661451), comparing TAVI with medical therapy in patients with moderate AS and systolic HF, will shed even more light on the optimal treatment strategy for patients with LFLG AS and reduced LVEF. ${ }^{29}$

\section{Limitations}

There are several limitations inherent in the present analysis in addition to its retrospective design. First, AVCd was quantified by using contrast-enhanced CT images. The presented sex-specific prognostic cut-offs for low AVCd may therefore underlie certain restrictions. Second, another limitation of this study is the lack of a medically treated control group of patients with LFLG AS. Future studies will have to verify our results by using non-contrast CT images and including medically treated patients with LFLG AS. Third, the main limitation of our study remains that DSE was not part of the routine assessment for LFLG AS severity. Hence, we cannot rule out that patients with pseudo-severe AS were included in the study population. Therefore, it might be possible that low AVCd represents a surrogate parameter of pseudo-severe AS and that its adverse impact on outcome could have been partially foreseen by unveiling pseudo-severe AS with DSE. However, as contractile reserve is only present in about half of patients with LFLG AS and reduced LVEF, DSE is often non-diagnostic and guidelinerecommended DSE cut-offs like peak stress Pmean $\geq 40 \mathrm{~mm} \mathrm{Hg}$ or peak stress AVA $\leq 1.0 \mathrm{~cm}^{2}$ have failed to predict outcome among patients with LFLG AS and reduced LVEF under medical treatment. ${ }^{3}{ }^{30}$ Moreover, there is accumulating data supporting that aortic valve replacement may be beneficial in patients with pseudosevere AS, which might further weaken the importance of DSE in patients with LFLG AS and reduced LVEF. ${ }^{31}$ Fourth, the inclusion of baseline laboratory parameters (ie, NT-proBNP or troponin) into score systems for risk prediction in patients with LFLG AS and reduced LVEF represents an interesting approach, which was not practicable for the present study.

\section{CONCLUSIONS}

Based on data from a large multicentre registry, the RELiEF TAVI score is a novel risk prediction tool for patients with LFLG AS and reduced LVEF scheduled for TAVI. The score consists of simple clinical, echocardiographic and CT parameters at baseline and may help identify patients with LFLG AS and reduced LVEF at high risk for mortality or cardiovascular events after TAVI. The RELiEF TAVI score could serve as an important tool to facilitate decision-making in this complex subset of patients with AS.

\section{Author affiliations}

${ }^{1}$ Department of Cardiology, University Heart and Vascular Center Hamburg, Hamburg, Germany

${ }^{2}$ German Centre for Cardiovascular Research (DZHK), Partner site Hamburg/Kiel/ Luebeck, Germany

${ }^{3}$ Department of Cardiology, Heart Center Bonn, University Hospital Bonn, Bonn, Germany

${ }^{4}$ Department of Cardiology, Heart Center, University of Cologne, Cologne, Germany ${ }^{5}$ Department of Internal Medicine III, Cardiology, Angiology, and Critical Care, University Hospital Schleswig-Holstein, Kiel, Germany

${ }^{6}$ Clinic for General and Interventional Cardiology/Angiology, Heart and Diabetes Center North Rhine-Westphalia, Bad Oeynhausen, Germany

${ }^{7}$ Department of Cardiovascular Surgery, University Heart and Vascular Center Hamburg, Hamburg, Germany

Twitter Sebastian Ludwig @SebLudwig1

Contributors SL and NS are responsible for the overall content. SL is acting as guarantor. SL and NS contributed to conceptualisation. SL, AG and NS contributed to methodology. AG contributed to formal analysis. SL, AG, MS, DW, J-MS, AS, MA, VM, DF, HS, TR, MP, LC, NS contributed to investigation, resources, data curation. SL and NS contributed to original manuscript preparation. AG, MS, DW, J-MS, AS, MA, VM, DF, HS, TR, MP, LC contributed to manuscript review and editing. SL and AG contributed to visualisation.

Funding The authors have not declared a specific grant for this research from any funding agency in the public, commercial or not-for-profit sectors.

Competing interests SL reports non-financial support from Edwards Lifesciences. MS reports non-financial support from Abbott Vascular, Biotronik, Boehringer Ingelheim, Boston Scientific, Edwards Lifesciences, Nicolai Medizintechnik, OrbusNeich Medical, personal fees from Abiomed, Amgen, AstraZeneca, Bayer Healthcare, Bristol-Myers Squibb, Boston Scientific, Medtronic, Philips and Shockwave Medical, grants from Philips. DW reports personal fees from AstraZeneca, Bayer, Berlin-Chemie and Novartis. J-MS is proctor for Medtronic and Boston Scientific, received research grants and speaker honoraria from Edwards Lifesciences, Medtronic and Boston Scientific. DF is consultant for Edwards Lifesciences and Medtronic and received research funding from Edwards Lifesciences. TR received speaker honoraria from Boston Scientific, Edwards, Medtronic, Abbott. LC is proctor and consultant for Edwards Lifesciences and Boston Scientific and consultant for Medtronic and Abbott Vascular. NS reports personal fees from Boston Scientific and non-financial support from Abbott and Edwards Lifesciences.

Patient consent for publication Consent obtained directly from patient(s).

Ethics approval This study involves human participants and was approved by Ethik-Kommission der Ärztekammer Hamburg, ID: PV4304 Participants gave informed consent to participate in the study before taking part.

Provenance and peer review Not commissioned; internally peer reviewed. Data availability statement Data are available upon reasonable request.

Open access This is an open access article distributed in accordance with the Creative Commons Attribution Non Commercial (CC BY-NC 4.0) license, which permits others to distribute, remix, adapt, build upon this work non-commercially, 
and license their derivative works on different terms, provided the original work is properly cited, appropriate credit is given, any changes made indicated, and the use is non-commercial. See: http://creativecommons.org/licenses/by-nc/4.0/.

\section{ORCID iDs}

Sebastian Ludwig http://orcid.org/0000-0002-5752-4951

Dirk Westermann http://orcid.org/0000-0002-7542-1956

Victor Mauri http://orcid.org/0000-0003-2678-3501

Derk Frank http://orcid.org/0000-0001-7561-075X

Hatim Seoudy http://orcid.org/0000-0001-9198-5965

\section{REFERENCES}

1 Reinöhl J, Kaier K, Reinecke $\mathrm{H}$, et al. Effect of availability of transcatheter aortic-valve replacement on clinical practice. $N$ Engl J Med 2015;373:2438-47.

2 Vahanian A, Beyersdorf F, Praz F, et al. 2021 ESC/EACTS guidelines for the management of valvular heart disease. Eur Heart $J$ 2021:ehab395.

3 Ribeiro HB, Lerakis S, Gilard M, et al. Transcatheter Aortic Valve Replacement in Patients With Low-Flow, Low-Gradient Aortic Stenosis: The TOPAS-TAVI Registry. J Am Coll Cardiol 2018;71:1297-308.

4 Herrmann HC, Pibarot P, Hueter I, et al. Predictors of mortality and outcomes of therapy in low-flow severe aortic stenosis: a placement of aortic transcatheter valves (partner) trial analysis. Circulation 2013;127:2316-26.

5 Lauten A, Figulla HR, Möllmann $\mathrm{H}$, et al. TAVI for low-flow, lowgradient severe aortic stenosis with preserved or reduced ejection fraction: a subgroup analysis from the German aortic valve registry (GARY). Eurolntervention 2014:10:850-9.

6 Puls M, Korte KP, Bleckmann A, et al. Long-term outcomes after TAVI in patients with different types of aortic stenosis: the conundrum of low flow, low gradient and low ejection fraction. Eurolntervention 2017;13:286-93.

7 Otto CM, Pearlman AS, Comess KA, et al. Determination of the stenotic aortic valve area in adults using Doppler echocardiography. J Am Coll Cardiol 1986; 7:509-17.

8 Ludwig S, Goßling A, Waldschmidt L, et al. TAVR for low-flow, low-gradient aortic stenosis: prognostic impact of aortic valve calcification. Am Heart J 2020;225:138-48.

9 Seiffert M, Fujita B, Avanesov M, et al. Device landing zone calcification and its impact on residual regurgitation after transcatheter aortic valve implantation with different devices. Eur Heart J Cardiovasc Imaging 2016;17:576-84.

10 WHO. Body mass index - BMI. Available: https://www.euro.who. int/en/health-topics/disease-prevention/nutrition/a-healthy-lifestyle/ body-mass-index-bmi [Accessed 8 Nov 2021]

11 Nashef SAM, Roques F, Sharples LD, et al. EuroSCORE II. Eur J Cardiothorac Surg 2012;41:734-45.

12 Clavel M-A, Magne J, Pibarot P. Low-gradient aortic stenosis. Eur Heart J 2016:37:2645-57.

13 Tang GHL, Verma S, Bhatt DL. Transcatheter aortic valve replacement in low-risk patients. Circulation 2019;140:801-3.

14 Amabile N, Agostini H, Gilard M, et al. Impact of low preprocedura transvalvular gradient on cardiovascular mortality following TAVI: an analysis from the France 2 registry. Eurolntervention 2014;10:842-9.

15 Dahou A, Magne J, Clavel M-A, et al. Tricuspid Regurgitation Is Associated With Increased Risk of Mortality in Patients With Low-
Flow Low-Gradient Aortic Stenosis and Reduced Ejection Fraction: Results of the Multicenter TOPAS Study (True or Pseudo-Severe Aortic Stenosis). JACC Cardiovasc Interv 2015;8:588-96.

16 Dahou A, Clavel M-A, Capoulade R, et al. Right ventricular longitudinal strain for risk stratification in low-flow, low-gradient aortic stenosis with low ejection fraction. Heart 2016;102:548-54.

17 Lindman BR, Zajarias A, Maniar HS, et al. Risk stratification in patients with pulmonary hypertension undergoing transcatheter aortic valve replacement. Heart 2015;101:1656-64.

18 Mok M, Nombela-Franco L, Dumont E, et al. Chronic obstructive pulmonary disease in patients undergoing transcatheter aortic valve implantation: insights on clinical outcomes, prognostic markers, and functional status changes. JACC Cardiovasc Interv 2013;6:1072-84.

19 Le Ven F, Freeman M, Webb J, et al. Impact of low flow on the outcome of high-risk patients undergoing transcatheter aortic valve replacement. J Am Coll Cardiol 2013;62:782-8.

20 Kataoka A, Watanabe Y, Kozuma K, et al. Prognostic impact of low-flow severe aortic stenosis in Small-Body patients undergoing TAVR: the OCEAN-TAVI registry. JACC Cardiovasc Imaging 2018;11:659-69.

21 Schymik G, Würth A, Bramlage P, et al. Long-Term results of transapical versus Transfemoral TAVI in a real world population of 1000 patients with severe symptomatic aortic stenosis. Circ Cardiovasc Interv 2015;8:e000761.

22 Voigtländer L, Twerenbold R, Schäfer U, et al. Prognostic Impact of Underweight (Body Mass Index $<20 \mathrm{~kg} / \mathrm{m}^{2}$ ) in Patients With Severe Aortic Valve Stenosis Undergoing Transcatheter Aortic Valve Implantation or Surgical Aortic Valve Replacement (from the German Aortic Valve Registry [GARY]). Am J Cardiol 2020;129:79-86.

23 Mattila K, Haavisto M, Rajala S. Body mass index and mortality in the elderly. Br Med J 1986;292:867-8.

24 Clavel M-A, Messika-Zeitoun D, Pibarot P, et al. The complex nature of discordant severe calcified aortic valve disease grading. J Am Coll Cardiol 2013;62:2329-38.

25 Cartlidge TR, Bing R, Kwiecinski J, et al. Contrast-Enhanced computed tomography assessment of aortic stenosis. Heart 2021;107:1905-11.

26 Clavel M-A, Burwash IG, Pibarot P. Cardiac imaging for assessing Low-Gradient severe aortic stenosis. JACC Cardiovasc Imaging 2017;10:185-202.

27 Milhorini Pio S, Bax J, Delgado V. How valvular calcification can affect the outcomes of transcatheter aortic valve implantation. Expert Rev Med Devices 2020;17:773-84.

28 Clavel M-A, Pibarot P, Messika-Zeitoun D, et al. Impact of aortic valve calcification, as measured by MDCT, on survival in patients with aortic stenosis: results of an international registry study. J Am Coll Cardiol 2014;64:1202-13

29 Spitzer E, Van Mieghem NM, Pibarot P, et al. Rationale and design of the transcatheter aortic valve replacement to UNload the left ventricle in patients with advanced heart failure (TAVR UNLOAD) trial. Am Heart J 2016;182:80-8.

30 Annabi M-S, Touboul E, Dahou A, et al. Dobutamine Stress Echocardiography for Management of Low-Flow, Low-Gradient Aortic Stenosis. J Am Coll Cardiol 2018;71:475-85.

31 Annabi M-S, Côté N, Dahou A, et al. Comparison of early surgical or transcatheter aortic valve replacement versus conservative management in low-flow, Low-Gradient aortic stenosis using inverse probability of treatment weighting: results from the TOPAS prospective observational cohort study. J Am Heart Assoc 2020;9:e017870. 